RESEARCH ARTICLE

\title{
Genetic Profiling of Methicillin-resistant Staphylococcus aureus in An African Hospital by Multiplex-PCR
}

\author{
Shuaibu Suleiman Adeiza, Josiah Ademola Onaolapo, Busayo Olalekan Olayinka \\ Department of Pharmaceutical Microbiology, Faculty of Pharmaceutical sciences, Ahmadu Bello University, Zaria, \\ Kaduna, Nigeria
}

\begin{abstract}
Objectives: Methicillin resistant Staphylococcus aureus (MRSA) nasal carriage often precedes infection. This work aims to genetically profile MRSA carriage of resistant genes (mecA, blaZ and tetM) and certain virulence genes (nuc, pvl, spa and scn).

Methods: Phenotypically confirmed Staphylococcus aureus were Screened for oxacillin resistance and assessed for $\beta$-lactamase production. Further confirmation of identity was performed by multiplex PCR of the 16SrRNA, mecA, tetM, pvl, nuc, spa and scn genes. DNA gel fingerprint analysis was carried out and the bands were clustered in a dendrogram.

Results: The study analysed 81 Staphylococcus aureus isolates for Methicillin resistance out of which 38 (46.9\%) were MRSA. The DNA extraction method was effective for all MRSA isolates evaluated. All phenotypic MRSA isolates (100\%) were positive for the $S$. aureus specific 16SrRNA gene confirming that these isolates were $S$. aureus strains. The nuc gene was detected in 19/38 (50\%) of the MRSA isolates, blaZ gene in $14 / 38(36.8 \%)$ and tetM in $18 / 38(47.4 \%)$.
\end{abstract}

Conclusions: The need for improved vigilance to recognize MRSA strains cannot be overemphasized. If MRSA control is not tracked, these strains will increase exponentially. J Microbiol Infect Dis 2020; 10(3):144-153.

Keywords: Methicillin resistant Staphylococcus aureus, PCR simulation, mecA, Electrophoretogram

\section{INTRODUCTION}

The hallmark of Methicillin resistant Staphylococcus aureus (MRSA) is the proliferation of antimicrobial resistance and MRSA nasal carriage often precedes infection [1]. The feat of this bacterium as a pathogen is directly linked to its range of virulence and immune evasion factors that enable the bacterium to dodge host defenses [2].

MRSA, as we have come to accept, brought about a tumultuous antibiotic's resistance era in hospital and community sceneries worldwide, owing to its increased intransigency to several $\beta$ lactam antibiotics engendered by the acquisition $m e c A$ gene that codes for the production of modified penicillin binding protein $2 \mathrm{a}$ (PBP2a) [3].
Nasal secretions are mixtures of proteins, sugars and salts, containing e.g. lysozyme and immunoglobulins ( $\lg A$ and $\lg G$ ), as well as defensins and complement proteins [4]. $S$. aureus is resistant to lysozyme due to the cell wall modifying enzyme. Expression of several factors, including staphylococcal protein A (spa) and chemotaxis inhibitory protein of $S$. aureus (CHIPS) can limit opsonization by binding to the immunoglobulins, changing conformation bacterial cells to one not recognized by neutrophils. CHIPS and the staphylococcal complement inhibitor (SCIN/scn) are innate immune modulators, known to interfere with the complement system [5]. Other virulent factors possessed by this organism includes Hemolysin and Panton-Valentine leucocidin cause killing of white blood cells and red blood cells [5].

Correspondence: Dr. Shuaibu Suleiman Adeiza, Department of Pharmaceutical Microbiology, Faculty of Pharmaceutical Sciences, Ahmadu Bello University, Zaria, Kaduna, Nigeria 
The most common housekeeping genetic marker is the 16SrRNA gene because it is present in all bacteria [6]. PCR amplification from these genetically conserved parts of the bacterial genome is a sturdy procedure for the identification.

Nasal carriage is important for endogenous infections and for transmission to other individuals, as colonization of extra nasal sites often originates from the nasal reservoir. In this regard, special attention was paid to methicillinresistant (MRSA) which has become a foremost issue during the past three decades [7].

Data on molecular characterization of methicillin resistant Staphylococcus aureus of nasal origin is scanty in this part of the country (North West), and there are only few documentations of the situation in Sokoto.

This work aims to genetically profile the MRSA carriage of resistant genes (mecA, blaZ and tetM) and some selected virulence genes (nuc, pvl, spa and scn).

\section{METHODS}

\section{Staphylococcus aureus Isolates}

This study was approved by Ethics Committee of Sokoto state Ministry of health (SMH/1580/V. IV). Eighty-one phenotypically confirmed $S$. aureus (Microgen Staph ID kit) isolates collected randomly from the anterior nares of Patients and Staff of State-owned hospitals in Sokoto state Nigeria were used in this study.

\section{Screening for Oxacillin resistance}

A standardized suspension (0.5 McFarland) of $S$. aureus isolates were prepared and streaked onto Oxacillin resistance screening agar base (ORSAB) medium pre-supplemented with 6 $\mu \mathrm{g} / \mathrm{ml}$ oxacillin and $4 \%$ sodium chloride and incubated at $35{ }^{0} \mathrm{C}$ for 24 hours. The emergent bluish colonies from the overnight cultures were considered methicillin resistant [8].

\section{Rapid chromogenic test for $\beta$-lactamase detection}

The production of $\beta$-lactamase enzymes was verified by the chromogenic cephalosporin method using nitrocephin impregnated sticks (beta-lactamase stick) (BR0066A, Oxoid® UK) according to the manufacturer's instructions. A typical pure colony of $S$. aureus was selected from the growth medium. The colonies of interest were touched with the impregnated end of the stick. The stick was then rotated to pick up a minute slice of pure colony and was observed for color changes within 10 minutes up to an hour. A color change from yellow to pink-red indicated positive $\beta$-lactamase test [9].

\section{Primer design}

The primer design was based on partial coding sequences of genes of interest obtained from GenBank/NCBI. The open reading frames of these sequences were identified and noted. The regions of the template sequence to be excluded and included in the design were marked and the primers were calculated using Primer3Plus $®$. The forward and reverse primer sequences obtained were compared with other sequences in NCBI primer blast to rule out overlaps. Primers were designed to nest in the nonhomologous regions, to certify specificity and allow species-specific amplifications; to further verify this, the sequences were evaluated in a virtual PCR simulation before ordering for its production.

\section{PCR simulation}

PCR simulation was executed using Snap Gene $^{\mathrm{TM}}$ software (version 1.1.3) to determine the efficiency and the validity of the expected amplicon sizes of the proposed primers before ordering (Assumption-free). The target gene nucleotide sequences were retrieved from Gene bank and used as a matrix on which the chosen primer sequence was evaluated. Upon evaluation, the operational primers yielded oligonucleotides, PCR fragment length and a virtual agarose gel with observed band showing the expected amplicon size [10].

\section{Primer reconstitution}

The received primers were centrifuged briefly (8000 rpm for $30 \mathrm{~s}$ ) before reconstitution to get all the lyophilized DNA to the bottom of the tube. 100 micromolar of each stock primer was prepared as described by the manufacturer. Furthermore 10 micromolar of the working stock concentration of each primer were prepared by diluting 10 microliters of each stock primer with 90 microliters of nuclease-free water in $1.5 \mathrm{ml}$ 
nuclease-free microtubes. The primers used in this study is shown in Table 1.

\section{DNA extraction (Lysis, precipitation and purification and elution)}

An overnight culture of each of the $S$. aureus isolates in brain-heart infusion broth was suspended in $1.5 \mu$ Eppendorf tube, centrifuged at $12000 \mathrm{rpm}$ for $5 \mathrm{~min}$. The supernatants were discarded into a disinfecting jar and the pellets were re-suspended in a $200 \mu \mathrm{l}$ nuclease-free water (molecular grade). To the suspension, 20 $\mu \mathrm{l}$ of proteinase $\mathrm{K}$ and $200 \mu \mathrm{l}$ Qiagen $^{\mathrm{TM}}$ lysis buffer was added to each tube and vortexed for $15 \mathrm{~s}$ and incubated at $56{ }^{\circ} \mathrm{C}$ for $20 \mathrm{~min}$. The tubes were then centrifuged and transferred to the Qiagen spin column in a $2 \mathrm{ml}$ collection tube. The whole content of the Eppendorf tubes was transferred into QIAamp ${ }^{\mathrm{TM}}$ spin column inserted into $2 \mathrm{ml}$ collection tube, centrifuged at $8000 \mathrm{rpm}$ for $30 \mathrm{~s}$. Five hundred microliter of buffer AW1 was added to the spin column and centrifuged again at 8000rpm for $1 \mathrm{~min}$ and the flow through was discarded. $200 \mu \mathrm{l}$ of buffer AW2 was added and centrifuged at $14000 \mathrm{rpm}$ for 3 minutes. The tubes were further centrifuged for $14000 \mathrm{rpm}$ for $1 \mathrm{~min}$ to avoid carryover of wash buffer. The spin column was transferred into a freshly labelled $1.5 \mu \mathrm{l}$ nuclease-free microtubes. To unbind the attached DNA from silica membrane, $100 \mu \mathrm{l}$ of Qiagen ${ }^{\mathrm{TM}}$ elution buffer was added to the silica membrane in the spin column, incubated at room temperature for $5 \mathrm{~min}$ and centrifuged at $8000 \mathrm{rpm}$ for 1 minute [11]. The flow through (extracted DNA) was capped and stored at -20 ${ }^{0} \mathrm{C}$ until required.

\section{Multiplex PCR for 16SrRNA, nuc, blaZ and tetM genes}

PCR was performed using the thermal cycling conditions as stated by Qiagen Research UK [11]. A master mix was prepared in an Eppendorf tube and the total volume was determined by the number of samples to be analyzed.

A PCR cocktail was prepared containing $12.5 \mu \mathrm{l}$ of Qiagen master mix. In accordance with the manufacturer's instruction (Qiagen), Lyophilized primers were reconstituted using molecular grade water and $0.25 \mu \mathrm{M}$ of each primer $(0.5 \mu \mathrm{l}$ of each primer pair, forward and reverse) was added to the cocktail. In addition, $3 \mu \mathrm{l}$ nuclease- free water and $2.5 \mu \mathrm{l}$ of Q-reagent was added to the cocktail. Overall, $20 \mu \mathrm{l}$ of the mixture was then introduced into nuclease-free microtubes (PCR tubes) containing $5 \mu \mathrm{l}$ of DNA template. The tubes were then transferred into Applied Biosystems ${ }^{\circledR} 9700$ thermocycler. The machine was programmed thus; an initial denaturation step at $94{ }^{\circ} \mathrm{C}$ for 15 minutes followed by 35 cycles of denaturation (at $94{ }^{\circ} \mathrm{C}$ for $30 \mathrm{~s}$ ), annealing $\left(57^{\circ} \mathrm{C}\right.$ for 1 minute and $\left.30 \mathrm{~s}\right)$ and extension $\left(72{ }^{\circ} \mathrm{C}\right.$ for 2 minutes). The reaction was completed by final extension time of 10 minutes at a temperature of $72{ }^{\circ} \mathrm{C}$.

\section{Multiplex PCR for mecA, pvl, spa and scn}

Multiplex PCR was performed for detection of $m e c A$, pvl, spa and scn genes specific using primers shown in Table 3.2. The PCR was performed in a final reaction volume of $25 \mu \mathrm{L}$ consisting of; $12.5 \mu \mathrm{l}$ of Qiagen ${ }^{\mathrm{TM}}$ master mix ,5 $\mu \mathrm{L}$ of DNA template, $2.5 \mu \mathrm{L}$ of Q-reagent, $0.5 \mu \mathrm{L}$ of each primer pair ( $2 \mu \mathrm{L}$ entirely) and $3 \mu \mathrm{l}$ nuclease-free water. DNA was amplified on a thermocycler (Applied Biosystems $\AA$ 9700), and PCR conditions were as follows: Initial denaturation for $15 \mathrm{~min}$ at $94{ }^{\circ} \mathrm{C}, 30$ cycles of denaturation for $10 \mathrm{~s}$ at $94{ }^{\circ} \mathrm{C}$, annealing for $30 \mathrm{~s}$ at $59{ }^{\circ} \mathrm{C}$, extension for $1 \mathrm{~min}$ at $72{ }^{\circ} \mathrm{C}$, and a final extension of $10 \mathrm{~min}$ at $72{ }^{\circ} \mathrm{C}$.

\section{Gel Electrophoresis}

The PCR product (Amplicons) was electrophoresed on a $1.5 \%$ agarose gel prestained with ethidium bromide. A 100 bp plus ladder was loaded in the in the first well using 10x Qiagen ${ }^{\mathrm{TM}}$ loading dye [12]. The agarose gel was transferred into Bio-Rad gel documentation device and viewed using a UV trans-illuminator. Gel-lane and band analysis as well as point to point regression analysis of the electrophoretogram was carried out using Bio$\operatorname{Rad}^{\mathrm{TM}}$ image lab software version 3.0.

\section{DNA Gel-fingerprint analysis}

Phylogenetic relatedness of MRSA strains was determined by analysis of DNA fingerprints via BioNumerics $^{\mathrm{TM}}$ version 7.6 software (Applied Maths, Kortrijk, Belgium). Normalization steps were involved in the analysis to certify suitable gel-to-gel banding pattern contrast. A procedure of "band scoring" identified bands in each lane that combine to make the fingerprint based on 
the threshold of stringency and optimization settings. The band positions in the electrophoretograms were standardized for variation from lane-to-lane and gel-to-gel. Subsequent processes were performed using the unweighted pair group method with arithmetic averages (UPGMA) and cluster analysis with dice coefficient (18).

\section{Statistical analysis}

All statistical analysis was conducted with the SAS version 9.4 software package (SAS Institute Inc., Cary, NC, USA).

\section{RESULTS}

We analysed 81 Staphylococcus aureus isolates for methicillin resistance out of which 38 (46.9\%) were MRSA. The DNA extraction method was effective for all 38 MRSA isolates evaluated, Figure $1 \mathrm{~A}$ shows electrophoretograms of multiplex PCR (M-PCR) assay for 16SrRNA (956 bp), nuc (421 bp), blaZ (216 bp), tetM (158 bp) genes of 38 MRSA strains. The assay showed (Table 2) that all 38 MRSA isolates $(100 \%)$ were positive for the $S$. aureus specific 16SrRNA gene, further confirming that these isolates were $S$. aureus strains. The nuc gene was detected in 19/38 (50\%) of the MRSA isolates, blaZ gene in $14 / 38(36.8 \%)$ and tetM in 18/38 (47.4\%).

DNA gel finger print analysis was carried out and the bands were clustered in a dendrogram using dice coefficient based on band pattern similarity and intensity with a minimum band tolerance of $3 \%$. As evident in Figure 2A, five clusters with a similarity of $70 \%$ were identified. Four profile groups (A, B, C, and E) were the most predominant among the population when compared to group D. Profile A was the most common with $34.2 \% \quad(n=13)$ of the isolates belonging to this group and this was followed by profiles $A(9.9 \%, n=8)$, and B, C with $7.4 \%(n=6)$ on each group. Group D was the least populated group with only 5 isolates (6.2\%). The profiles of the groups are as follows; $A=16 \operatorname{SrRNA}+$ tetM 16SrRNA + nuc + tetM $(\mathrm{n}=8), \quad \mathrm{B}=16 \mathrm{SrRNA}+$ blaZ + tetM /16SrRNA+nuc + blaZ +tetM $(\mathrm{n}=6)$, $\mathrm{C}=16 \mathrm{SrRNA}+$ nuc /16SrRNA + nuc + blaZ $(\mathrm{n}=6), \quad \mathrm{D}=16 \mathrm{SrRNA}+$ tetM /16SrRNA + blaZ + tetM $(\mathrm{n}=5)$ and $\mathrm{E}=16 \mathrm{SrRNA}$ only/ 16SrRNA + blaZ $(n=13)$.
Figure 1B shows the electrophoretogram of multiplex PCR amplicons mecA (533 bp), pvl (433 bp), spa (350 bp), scn (130 bp) for 38 MRSA. The mecA gene was detected in 16 isolates, $p v /$ in 17 isolates, spa 18 isolates and scn in 17 isolates. Table 2 summarizes the distribution of amplified genes across the MRSA isolates. The dendrogram clustered five major clades at $70 \%$ similarity (Figure $2 \mathrm{~B}$ ). The five clades were designated by $A-E$. Clade $E$ was the most populated with $26.3 \%$ of the isolates and this was followed by profiles A, B, D and C with $23.7 \%, 18.4 \%, 18.4 \%$ and $10.5 \%$ of the MRSA isolates respectively. The profiles of the groups are as follows; Group $A=9$ isolates (were positive for spa, spa+scn only, pvl $+s c n$ ), Group $\mathrm{B}=7$ isolates $(m e c A, \operatorname{mec} A+p v l,+\operatorname{mec} A$ + spa), $\mathrm{C}=4$ isolates (predominantly mecA-scn positive isolates), $D=7$ isolates (in addition to being mecA positive, were predominantly $\mathrm{pvl}$ positive isolates), $\mathrm{E}=10$ isolates (predominantly $p v l$ and spa positive isolates) and a singleton (pvl, spa, scn).

\section{DISCUSSION}

All 38 MRSA isolates were positive for 16SrRNA genes and $42.1 \%(16 / 38)$ of the MRSA isolates carried the mecA gene, with 22 isolates not expressing the mecA gene. The 16SrRNA gene, the nuc gene and mecA gene PCR was used to identify and confirm isolates as MRSA. Elhassan et al [15] explained that the inability to detect $m e c A$ gene in some studies may not be unconnected with the fact that some $m e c A$ containing isolates might have lost the gene on prolong storage and probably due to higher temperatures between the preliminary characterization to the time of final molecular characterization as a result of inconsistent power supply in the environment as supported by some studies.

$\beta$-lactam resistance is a significant phenotype of MRSA and the amount of $\beta$-lactam resistance gene harbored and expressed differ among strains. The blaZ gene is a common $\beta$-lactam (penicillinase) resistant mechanism [16]. In this study $53.1 \%$ of the isolates were beta-lactamase positive, but not all the MRSA were positive for the beta-lactam resistance gene (36.8\%). A higher rate of $94.2 \%$ has been reported by (20) in contrast with our documentation. 
Table 1.Nucleotide sequences of Primer used in PCR.

\begin{tabular}{|c|c|c|c|c|c|}
\hline Gene & Primer & & Sequence (5'-->3') & Product & Accession \\
\hline \multirow[t]{2}{*}{ Housekeeping genetic marker } & 16SrRNA & $\mathrm{F}$ & AGGCAGCAGTAGGGAATCTT & 956 bp & LC416382 \\
\hline & & $\mathrm{R}$ & CCCGTCAATTCCTTTGAGTT & & \\
\hline \multirow[t]{2}{*}{ Thermostable nuclease } & nuc & $\mathrm{F}$ & GGCATATGTATGGCAATTGTTT & $421 \mathrm{bp}$ & DQ507381 \\
\hline & & $\mathrm{R}$ & ATACGCTAAGCCACGTCCAT & & \\
\hline \multirow[t]{2}{*}{$\beta$-lactam resistance (penicillinase) } & blaZ & $\mathrm{F}$ & GCTTCAACTTCAAAAGCGATAA & 216 bp & KY020077 \\
\hline & & $\mathrm{R}$ & TTGATTCCACCGATTTCGT & & \\
\hline \multirow[t]{2}{*}{ Tetracycline resistance } & tetM & $\mathrm{F}$ & TCGAGGTCCGTCTGAACTTT & 158 bp & AY452663 \\
\hline & & $\mathrm{R}$ & TGCAAAATAACAATTTCTCCAG & & \\
\hline \multirow[t]{2}{*}{ Methicillin resistance } & $\operatorname{mec} A$ & $\mathrm{~F}$ & TGGTAAAGGTTGGCAAAAAGA & 533 bp & KY788636 \\
\hline & & $\mathrm{R}$ & TTGTCCGTAACCGGAATCA & & \\
\hline \multirow[t]{2}{*}{ Panton Valentine leucocidin (Toxin) } & $p v l$ & $\mathrm{~F}$ & TAAGGGCAAACACTTGTGGA & $433 \mathrm{bp}$ & HQ020533 \\
\hline & & $\mathrm{R}$ & CCATTTGATCAAGACGAGCA & & \\
\hline \multirow[t]{2}{*}{ Staphylococcal Protein A (Toxin) } & spa & $\mathrm{F}$ & CTCAAGCACCAAAAGAGGAAG & variable & EF203507 \\
\hline & & $\mathrm{R}$ & ATGTACTCCGTTGCCGTCTT & & \\
\hline \multirow[t]{2}{*}{ Staphylococcal complement inhibitor } & scn & $\mathrm{F}$ & ACTTGCGGGAACTTTAGCAA & 130 bp & LT992473 \\
\hline & & $\mathrm{R}$ & GCATACATTGCTTTTTGACCTG & & \\
\hline
\end{tabular}

Table 2. Distribution of amplified genes across 38 phenotypic MRSA Isolates

\begin{tabular}{llll}
\hline Amplicons & & Frequency $(\mathrm{n})$ & Percentage $(\%)$ \\
\hline 16SrRNA & Positive & 38 & 100 \\
nuc & Negative & 19 & 50 \\
& Positive & 19 & 50 \\
blaZ & Negative & 24 & 63.2 \\
& Positive & 14 & 36.8 \\
tetM & Negative & 52.6 \\
& Positive & 20 & 47.4 \\
mecA & Negative & 18 & 57.9 \\
& Positive & 22 & 42.1 \\
Pvl & Negative & 16 & 55.3 \\
& Positive & 21 & 44.7 \\
Spa & Negative & 17 & 52.6 \\
& Positive & 20 & 47.4 \\
Scn & Negative & 18 & 55.3 \\
& Positive & 21 & 44.7 \\
\hline
\end{tabular}

The difference carriage could probably be ascribed to strain diversity difference in laboratory methodologies. The dual presence blaZ and mecA genes among the strains agree with previous reports that the $\beta$-lactam resistance mechanism mediated by mecA keeps the bla systems active (23). In this regard, the absence of blaZ gene among some MRSA positive strains in this study may be associated to the presence of other variants of the bla genes not investigated in this study. 


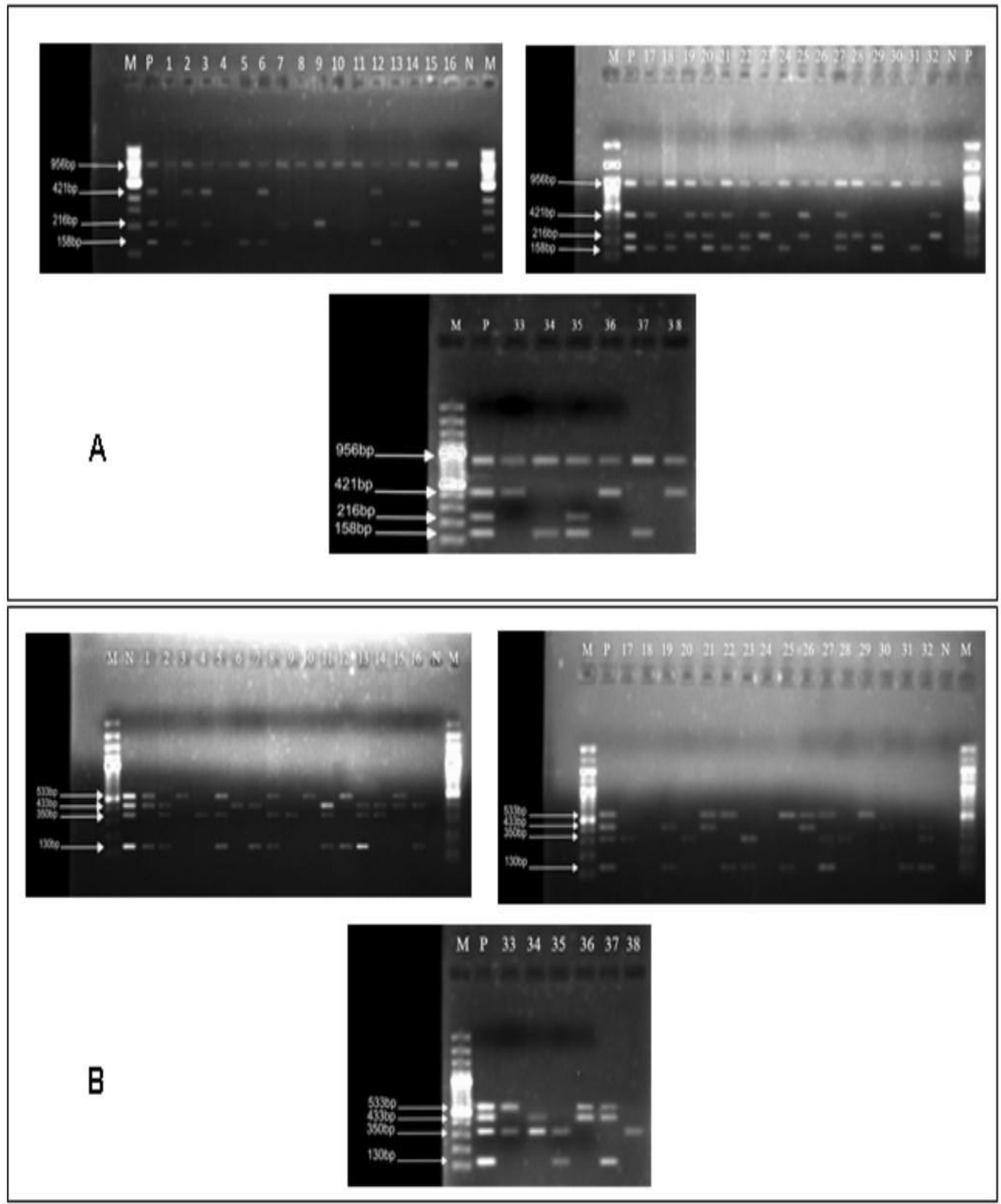

Figure 1. (A) Electrophoretogram of ethidium bromide stained gel of $38 \mathrm{Nasal}$ swab isolates showing amplification of 16SrRNA (956 bp), nuc (421 bp), blaZ (216 bp), tetM (158 bp) Genes for identification and characterization of Staphylococcus aureus. In the figure, M is $100 \mathrm{bp}+$ DNA ladder, $\mathrm{P}$ and $\mathrm{N}$ is Positive and Negative control. (B) Amplification multiplex PCR products of mecA (533 bp), pvl (433 bp), spa (350 bp), scn (130 bp) gene for identification of MRSA and its virulence characteristics. In the figure $\mathrm{M}$ is $100 \mathrm{bp}+\mathrm{DNA}$ ladder. $\mathrm{P}$ and $\mathrm{N}$ represents positive and negative controls. 
A



B

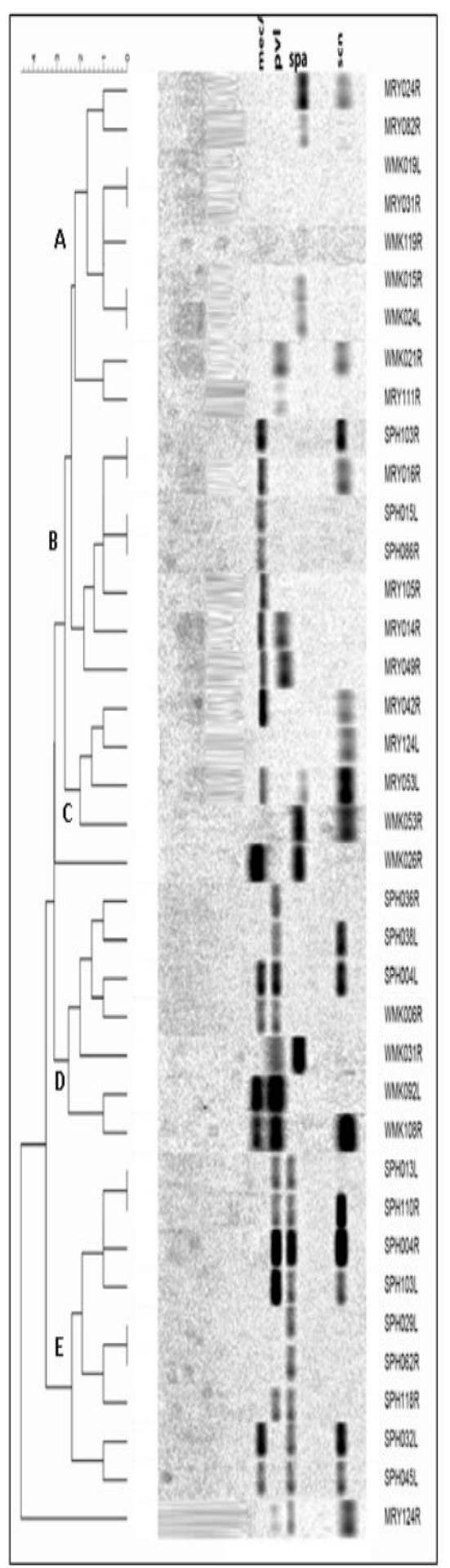

Figure 2. Dendrogram representing the genetic relatedness of 38 methicillin resistant $S$. aureus in terms of presence of (A) 16srRNA, nuc, blaZ and tetM genes (B) mecA, pvl, spa, scn amplicons. Strain designations are specified on the right end of the dendrogram. Cluster analyses was performed with dice comparison settings and an unweighted pair group method with arithmetic mean dendrogram type. The scale bar at the top of the dendrogram represents percentage similarity. 
Tetracycline resistance genes when expressed, confer resistance by binding to the ribosome and clearing the drug from its binding site. The most studied and prevalent tetracycline resistance determinants are tetM and tetO [21]. We screened all MRSA strains for tetM because the ribosomal protection protein is the most widely distributed of the two and earlier studies have identified it in 80 different genera (39 gramnegative and 41 gram-positive)[22]. Of the screened strains, $47.4 \%(18 / 38)$ was tetM positive. This observed rate is consistent with $49 \%$ reported by [18]and lesser than $63.6 \%$ reported by [23]. The reported rates may be due to the difference in sample population, or increased acquisition of tet $M$ in recent years [24]. Dual carriage of mecA and tetM genes among MRSA isolates reported in this study is less than $57.1 \%$ reported by (27). This may have probably arisen because both genes might be located on the same or associated genetic element.

scn is a potent antigen that evokes high antibody titers in $S$. aureus-colonized individuals. It helps the $S$. aureus to invade compliment systems of the human immune system. This molecular marker for livestock association was amplified in $44.7 \%$ of MRSA isolates in this study. Its absence in 55.3\% suggests the possibility of zoonotic origin (Livestock associated MRSA) [26]. A previous study by [27] reported a higher rate of $95 \%$ carriage among MRSA isolates.

Panton Valentine leucocidin increases the pathogenicity of $S$. aureus by fast-tracking cell decay and death, thus contributing to morbidity and mortality [28]. pvl is used as a marker for community-acquired MRSA. i.e. it differentiates hospital-acquired MRSA (HA-MRSA) and community-acquired MRSA (CA-MRSA). Reports from various countries show the rise prevalence of $p v l$ among MRSA isolates. The high prevalence of the pvl (44.7\%) gene detected in this study corroborates $48.7 \%$ carriage rate reported by a previous study [27]. The carriage of $p v l$ gene varies greatly between geographical locations and populations. The reason for the observed difference is largely unknown. The variability in number of virulence genes in this study was not unexpected because these genes are subjected to variable selective pressures.

In this study, 20 isolates of Staphylococcus aureus $(52.6 \%)$ had no spa gene, similar result was observed in a study by [29], in which spa gene was absent in $58.3 \%$ of patients. The absence could be due to deletion or addition mutations by which nucleotides are inserted or deleted. These sorts of mutation may have hindered the primer used during the PCR reaction circles from binding to the modified nucleotide resulting in a non-spa typeable strain[30]. Most proteins are usually bound to the bacterial cell wall; however, during the stationary phase of bacteria growth some $S$. aureus strains have been known to release protein-A into the surrounding medium due to autolysis [30]. This phenomenon may have contributed to prevalent spa-negative strains found in this study.

A drawback of our study was the few number of $S$. aureus investigated which may not have given a true picture of various genetic distribution. However, our findings stress the need for improved MRSA surveillance in the study centers.

\section{Conclusion}

We documented genetic methicillin resistance in this study by the successful detection of 16SrRNA and mecA gene among phenotypic MRSA isolates. The virulence genes reported here brings to light pathogenic propensities of MRSA. Existence of tetM genes confirms that besides being methicillin resistant, the isolates were tetracycline resistant. The results suggest the likelihood that infections caused by MRSA exists in our study center. We cannot overemphasise the need for improved vigilance to recognize MRSA strains. These strains will increase exponentially if we do not control MRSA spread.

\section{ACKNOWLEDGMENTS}

We are obliged to Mr. Abdulmalik B.S and Mrs. Halima Salihu of Usmanu Danfodiyo University Sokoto for their technical assistance.

Declaration of Conflicting Interests: The authors declare that they have no conflict of interest.

Financial Disclosure: No financial support was received. 


\section{REFERENCES}

1. Alexander JAN, Chatterjee SS, Hamilton SM, et al. Structural and kinetic analyses of penicillin-binding protein 4 (PBP4)-mediated antibiotic resistance in Staphylococcus aureus. Journal of Biological Chemistry 2018; 293: 19854-19865.

2. Frank DN, Feazel LM, Bessesen MT, et al. The human nasal microbiota and Staphylococcus aureus carriage. PloS one 2010; 5: e10598.

3. Akgül Ö, Çetin Y, Bora G, et al. Evaluation of $M e c A$ Gene Occurrence of Gram-Positive Bacteria Isolated from Patients with Otitis. Microbiology Research Journal International 2018; 1-8.

4. Bagnoli F, Rappuoli R, Grandi G. Staphylococcus aureus: Microbiology, Pathology, Immunology, Therapy and Prophylaxis. Springer, 2018.

5. McCarthy AJ, Lindsay JA. Genetic variation in Staphylococcus aureus surface and immune evasion genes is lineage associated: implications for vaccine design and host-pathogen interactions. BMC microbiology 2010; 10: 173.

6. Manjul AS, Shirkot P. 16S rRNA gene sequencing for bacterial identification of pullulanase synthesizing thermophilic bacteria contributing to big data. IJCS 2018; 6: 2769-2773.

7. Singh G, Broor S, Agarwal P. Molecular characterisation of Staphylococcus aureus using spa typing as a diagnostic tool in Haryana, India. Indian journal of medical microbiology 2018; 36: 26.

8. Aklilu E, Hui Ying C. First mecC and mecA Positive Livestock-Associated Methicillin Resistant Staphylococcus aureus (mecC MRSA/LA-MRSA) from Dairy Cattle in Malaysia. Microorganisms 2020; 8: 147.

9. Dilnessa T, Bitew A. Prevalence and antimicrobial susceptibility pattern of methicillin resistant Staphylococcus aureus isolated from clinical samples at Yekatit 12 Hospital Medical College, Addis Ababa, Ethiopia. BMC infectious diseases 2016; 16: 398.

10. [Antonio L, Simon JP, Jegathese O, et al. Making Inexpensive Insulin Using Microalgae. Experiment Moving Science Forward, https://experiment.com/projects/support-our-efforts-todemonstrate-that-insulin-and-oral-vaccines-can-becheaply-made-in-microalgae/methods (2016, accessed 30 May 2020).

11. Bien H. QIAgen DNeasy Blood \& Tissue kit, cultured cells. Epub ahead of print 10 September 2015. DOI: $10.17504 /$ protocols.io. dsz6f5.

12. Giske CG, Dyrkell F, Arnellos D, et al. Transmission events and antimicrobial susceptibilities of methicillin-resistant Staphylococcus argenteus in
Stockholm. Clinical Microbiology and Infection 2019; 25: 1289-e5.

13. Cha W, Mosci RE, Wengert SL, et al. Comparing the Genetic Diversity and Antimicrobial Resistance Profiles of Campylobacter jejuni Recovered from Cattle and Humans. Front Microbiol; 8. Epub ahead of print 2017. DOI: 10.3389/fmicb.2017.00818.

14. Velázquez-Guadarrama N, Olivares-Cervantes $A L$, Salinas $E$, et al. Presence of environmental coagulase-positive staphylococci, their clonal relationship, resistance factors and ability to form biofilm. Revista Argentina de Microbiología 2017; 49: 15-23.

15. Elhassan MM, Ozbak HA, Hemeg HA, et al. Absence of the mecA gene in methicillin resistant Staphylococcus aureus isolated from different clinical specimens in shendi city, Sudan. BioMed research international; 2015.

16. Akpaka PE, Roberts R, Monecke S. Molecular characterization of antimicrobial resistance genes against Staphylococcus aureus isolates from Trinidad and Tobago. Journal of Infection and Public Health 2017; 10: 316-323.

17. Vali L, Dashti AA, Mathew $F$, et al. Characterization of Heterogeneous MRSA and MSSA with Reduced Susceptibility to Chlorhexidine in Kuwaiti Hospitals. Frontiers in Microbiology; 8. Epub ahead of print 20 July 2017. DOI: 10.3389/fmicb.2017.01359.

18. Thong K, Hanifah $\mathrm{Y}$, Lim $\mathrm{K}$, et al. ermA, ermC , tet $M$ and tetK are essential for erythromycin and tetracycline resistance among methicillin-resistant Staphylococcus aureus strains isolated from a tertiary hospital in Malaysia. Indian Journal of Medical Microbiology 2012; 30: 203.

19. Liu P, Xue H, Wu Z, et al. Effect of bla regulators on the susceptible phenotype and phenotypic conversion for oxacillin-susceptible $\operatorname{mec} A$-positive staphylococcal isolates. Journal of Antimicrobial Chemotherapy 2016; 71: 2105-2112.

20. Milheiriço C, Portelinha A, Krippahl L, et al. Evidence for a purifying selection acting on the $\beta$ lactamase locus in epidemic clones of methicillinresistant Staphylococcus aureus. BMC microbiology 2011; 11: 76 .

21. Dönhöfer A, Franckenberg S, Wickles $S$, et al. Structural basis for TetM-mediated tetracycline resistance. PNAS 2012; 109: 16900-16905.

22. Roberts MC. Tetracyclines: Mode of Action and their Bacterial Mechanisms of Resistance. In: Bonev BB, Brown NM (eds) Bacterial Resistance to Antibiotics - From Molecules to Man. Wiley, pp. 101124.

23. Emaneini $M$, Bigverdi $R$, Kalantar $D$, et al. Distribution of genes encoding tetracycline resistance and aminoglycoside modifying enzymes in 
Staphylococcus aureus strains isolated from a burn center. Ann Burns Fire Disasters 2013; 26: 76-80.

24. Worthing KA, Abraham S, Coombs GW, et al. Clonal diversity and geographic distribution of methicillin-resistant Staphylococcus pseudintermedius from Australian animals: discovery of novel sequence types. Veterinary microbiology 2018; 213: 58-65.

25. Jones RN, Ross JE, Castanheira M, et al. United States resistance surveillance results for linezolid (LEADER Program for 2007). Diagnostic Microbiology and Infectious Disease 2008; 62: 416-426.

26. Eko KE, Forshey BM, Carrel M, et al. Molecular characterization of methicillin-resistant Staphylococcus aureus (MRSA) nasal colonization and infection isolates in a Veterans Affairs hospital. Antimicrob Resist Infect Control 2015; 4: 10.

27. Senok A, Ehricht R, Monecke S, et al. Molecular characterization of methicillin-resistant Staphylococcus aureus in nosocomial infections in a tertiary-care facility: emergence of new clonal complexes in Saudi Arabia. New Microbes and New Infections 2016; 14: 13-18.

28. Calfee DP. Trends in Community Versus Health Care-Acquired Methicillin-Resistant Staphylococcus aureus Infections. Current infectious disease reports 2017; 19: 48.

29. Al-Ruwaili MA. The coa, mec, and spa Genes Diversity among Methicillin-resistant Staphylococcus aureus Strains from Health-care Workers and Patients. Nigerian Journal of Clinical Practice | 2018; 21: $1065-1074$.

30. Rigi G, Ghaedmohammadi S, Ahmadian G. A comprehensive review on staphylococcal protein $A$ (Spa): Its production and applications. Biotechnology and Applied Biochemistry 2019; 66: 454-464. 